\title{
ИЛЛЮСТРАЦИЯ ЛОКАЛЬНЫХ РЕЛАКСАЦИОННЫХ ФАЗОВЫХ ПЕРЕХОДОВ В АНИЗОТРОПНЫХ ПРИМЕСНЫХ ЦЕНТРАХ
}

\author{
(Представлена В. Хижняковым)
}

Показано, что при варьировании параметра, изменяющего вероятность переориентации невзаимодействующих анизотропных примесных центров, возможно наблюдение явлений типа локальных релаксационных фазовых переходов. Среднее искажение решетки и расщепление динамического резонанса от активных колебаний ведут себя, как параметры порядка. Анализируются временные эффекты.

В последнее время значительно возрос интерес к проблеме локальных фазовых переходов и родственных им физических явлений $\left[{ }^{1-3}\right]$. Аналогично двум типам структурных фазовых переходов в кристаллах (смещения и ориентационного упорядочения) можно выделить две возможности собственного локального понижения симметрии примесного центра [ $\left.{ }^{1}\right]$. При варьировании в критических пределах физического параметра, являющегося решающим для возникновения устойчивой анизотропии как таковой в отдсльном центре, возникает аналог перехода типа смещения (ом., напр., $\left.\left[{ }^{3}\right]\right)$. Другой путь заключается в установлении ориентационной упорядоченности аннзотропных центров, слу . жащей оснсвой для локальных фазовых переходов релаксационного типа. Ввиду автоматического наличия ориентационно вырожденных низкосимметричных конфигураций у анизотропного центра, локальный фазовый переход, связанный с усреднением по этим конфигурациям, должен быть достаточно универсальным свойством таких систем. Применение понятий фазового перехода позволяет здесь взглянуть на достаточно долго разрабатываемую область не совсем с традиционных позиций.

При одинаковой заселенности ориентационно вырожденных низкосимметричных конфигураций центр эффективно изотропен. Для наблюдения анизотропных свойств необходима различная заселенность этих конфигураций в течение характеристического времени эксперимента * $t_{\text {э. }}$ Отдельная низкосимметричная конфигурация принципиально нестационарна в связи с туннелированием между ориентационно вырожденными конфигурациями. Если соответствующее время релаксации $\tau<t_{3}$, анизотропные свойства центра в данном эксперименте как таковые не обнаруживаются (наличие процессов релаксации, однако, может быть выявлено). С изменением физического параметра, меняющего $\tau$,

* Это обстоятельство может быть причиной интересных эффектов также около температур фазовых переходов типа смещения самих кристаллов [ $\left.{ }^{4}\right]$. 
при фиксированном $t_{5}$ возможно наблюдение фазового перехода релаксационного типа, наступающего при $\tau \sim t_{3}$. Ниже мы пронллюстрируем это более подробно на простой модели и покажем, что физические параметры, отображающие анизотропные свойства центра, ведут себя достаточно типично для параметра порядка фазового перехода.

Пусть в кубическом кристалле имеются невзаимодействующие примесные центры, анизотропия которых возникла вследствие «вмораживания» низкосимметричного искажения структуры по компонентам $Q_{x}, Q_{y}, Q_{z}$ колебательной квазимолекулярной моды взанмодействия симмегрии $F_{1 u}\left[{ }^{5}\right]$. Имеются шесть низкосимметричных конфигураций типа $Q_{z}^{2}=Q_{z 0}^{2} ; Q_{y 0}=Q_{x 0}=0$, числа заполнения которых обозначим через $n_{ \pm i}, i=x, y, z, \sum_{i} n_{ \pm i}=1$. Будем считать, что к моменту $t=0$ приготовлено состояние $n_{z}=1$. Частота активных колебаний около каждой низкосимметричной конфигурации будет расщеплена вследствие ее тетрагональной симметрии, так что соответствующие гармонические части потенциальной энергии могут быть записаны в виде

$$
\begin{aligned}
& F_{ \pm z}=\alpha\left(Q_{z} \mp Q_{z 0}\right)^{2}+\beta\left(Q_{x}^{2}+Q_{y}^{2}\right), \\
& F_{ \pm x}=\alpha\left(Q_{x} \mp Q_{x 0}\right)^{2}+\beta\left(Q_{y}^{2}+Q_{z}^{2}\right), \\
& F_{ \pm y}=\alpha\left(Q_{y} \mp Q_{y 0}\right)^{2}+\beta\left(Q_{x}^{2}+Q_{z}^{2}\right),
\end{aligned}
$$

при этом $Q_{x 0}{ }^{2}=Q_{y 0}{ }^{2}=Q_{z 0}{ }^{2}$. Величины $\alpha$ и $\beta$ определяются конкретной моделью. Например, для потенциала

$$
E=-\frac{\varepsilon}{2}\left(1+\frac{4 V^{2}}{\varepsilon^{2}} \sum_{i} Q_{i}^{2}\right)^{1 / 2}+\frac{M \omega_{0}^{2}}{2} \sum_{i} Q_{i}^{2}+A \sum_{i, j}^{\prime} Q_{i}^{2} Q_{j}^{2},
$$

где $\varepsilon$ - энергетическое расстояние между электронными уровнями,

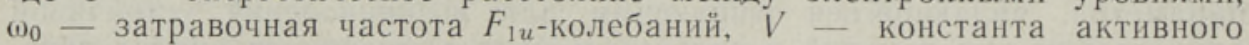
электрон-фононного взаимодействия, имеем

$$
\begin{aligned}
& \alpha=\frac{M \omega_{0}^{2}}{2}\left[1-\frac{\varepsilon^{2}\left(M \omega_{0}^{2}\right)^{2}}{4 V^{4}}\right], \quad \beta=A Q_{z 0}^{2}, \\
& Q_{z 0}^{2}=\frac{V^{2}}{\left(M \omega_{0}^{2}\right)^{2}}-\frac{\varepsilon^{2}}{4 V^{2}} .
\end{aligned}
$$

Потенциал (2) получается, например, при смешивании $A_{1 g}$ и $F_{1 u}$ электронных состояний колебаниями $F_{1 u}$ в ситуации псевдоэффекта ЯнаТеллера при учете собственного ангармонизма (константа $A$ ) активной моды $\left[{ }^{5,6}\right]$.

Потенциальную энергию анизотропного центра в процессе релаксации можно записать в виде

$$
F=\sum_{i} n_{ \pm i} F_{i}
$$

Вся система тождественных анизотропных центров будет давать линейные по их концентрации эффекты в полную динамику кристалла $\left(Q_{i}\right.$ являются комбинациями нормальных коөрдинат кристалла с дефектом).

Примем, что релаксация между низкосимметричными конфигурациями идет лишь $90^{\circ}$-путем (т. е. нет прямых переходов между конфигурациями сс $+Q_{i 0}$ и $\left.-Q_{i 0}\right)$ с верюятностью $W$. Тогда 


$$
\begin{aligned}
& n_{z}=1 / 6\left(1+3 \mathrm{e}^{-4 W t}+2 \mathrm{e}^{-6 W t}\right), \\
& n_{-z}=1 / 6\left(1-3 \mathrm{e}^{-4 W t}+2 \mathrm{e}^{-6 W t}\right), \\
& n_{x}=n_{-x}=n_{y}=n_{-y}=1 / 6\left(1-\mathrm{e}^{-6 W t}\right) .
\end{aligned}
$$

Вычисляя (5), получаем

$$
F=k_{\|}\left(Q_{z}-\bar{Q}_{z 0}\right)^{2}+k_{\perp}\left(Q_{x}^{2}+Q_{y}^{2}\right)+\alpha Q_{z 0}^{2}-k_{\|} \bar{Q}_{z 0}^{2},
$$

где

$$
\begin{aligned}
& k_{\|}=\alpha\left(n_{z}+n_{-z}\right)+4 \beta n_{x}, \\
& k_{\perp}=2(\alpha+\beta) n_{x}+\beta\left(n_{z}+n_{-z}\right), \\
& \bar{Q}_{z 0}=\alpha / k_{\|}\left(n_{z}-n_{-z}\right) Q_{z 0} .
\end{aligned}
$$

Величина $\bar{Q}_{z 0}$ представляет собой среднее измеряемое низкосимметричное искажение центра и обнаруживает в процессе выравнивания $n_{ \pm i}$ поведение, характерное для параметра порядка (при $n_{z}=1$ имеет место $\bar{Q}_{z 0}=Q_{z 0}$, при $n_{ \pm i}=1 / 6$ соответственно $\left.\bar{Q}_{z 0}=0\right)$. Исчезновение наведенной в системе анизотропии отображает также расщепление

$$
k_{\|}-k_{\perp}=(\alpha-\beta)\left(n_{z}+n_{-z}-2 n_{x}\right),
$$

причем для $n_{z}=1, k_{\|}=\alpha, k_{\perp}=\beta ;$ для $n_{ \pm i}=1 / 6, \quad k_{\|}=k_{\perp}=k_{0}=1 / 3(\alpha+$ $+2 \beta)$, a $k_{\|}-k_{0}=2 / 3\left(k_{\|}-k_{\perp}\right), k_{\perp}-k_{0}=-1 / 3\left(k_{\|}-k_{\perp}\right)$.

На основании (7) имеем уравнения движения $\left(Y=Q_{z}-\bar{Q}_{z 0}\right.$, $\left.Q_{\perp}=Q_{x, y}\right)$

$$
\begin{aligned}
& \ddot{Y}=-2 k_{\|}(t) Y, \\
& \ddot{Q}_{\perp}=-2 k_{\perp}(t) Q_{\perp} .
\end{aligned}
$$

Можно показать (см. Приложение), что в интервале времени $\Delta t \ll W^{-1}$ около $t_{0}$ величины $k_{\|}\left(t_{0}\right)$ и $k_{\perp}\left(t_{0}\right)$ определяют частоты гармонического изменения активных квазимолекулярных координат. Оценки показывают, что даже в неблагоприятных случаях $\Delta t$ составляет $10^{2}-10^{3}$ периодов колебаний решетки. Это означает, что опыт, в котором время взаимодействия экспериментального устройства с кристаллом достаточно мало $t_{\text {э }} \ll W^{-1}$, позволит выявить по прошествии времени $t_{0} \mathrm{c}$ начала релаксации полностью упорядоченной системы сопровождающее снижение анизотропии уменьшение расщепления частот $\sqrt{2 k_{\|}\left(t_{0}\right)}-$ $-\sqrt{2 k_{\perp}\left(t_{0}\right)}$. В условиях, когда этим квазимолекулярным частотам можно сопоставить резонансы в фононном спектре $\left[{ }^{5}\right]$, их расщепление ведет себя, как параметр порядка. Подчеркнем, что мы рассматриваем динамическую ситуацию для каждого центра, когда имеет место описанное изменение колебательных частот. В статическом же случае ансамбля центров, локализованных в определенных низкосимметричных конфигурациях, должны наблюдаться две частоты, определяемые $\alpha$ и $\beta$, а изменение распределения центров должно приводить к изменению интенсивностей соответствующих резонансов.

Сами же резонансы в данном случае (ср. с [ [3]), вообе говоря, не являются мягкими, т. е. их частоты не обращаются в нуль при $n_{ \pm i}=1 / 6$. Мягкими свойствами должна обладать мода, отвечающая переориентации центров. Однако, в отличие от истинного фазового перехода, $\bar{Q}_{z 0}$ и 
$\left(k_{\|}-k_{\perp}\right)$ стремятся с физическим параметром, обусловливающим рост $W$, к нулю асимптотически, хотя и резко. Из выражений (9) и (6) для низкосимметричного искажения решетки следует $\left(Y_{0}=\alpha^{-1} Q_{z 0}^{-1} \bar{Q}_{z 0}\right)$

$$
\ddot{Y}_{0}=-\Omega^{2}(t) Y_{0},
$$

где

$$
\Omega^{2}(t)=8 W^{2}\left[1+\xi(t)-4 \xi^{2}(t)\right], \quad \xi=k_{\perp} / k_{\|} .
$$

В процессе выравнивания $n_{ \pm i}$, при $\xi_{k}=1 / 8(1+\sqrt{17}), \Omega^{2}$ обращается в нуль (точка перегиба), а в дальнейшей области стремления $\xi$ к 1 нарастают отрицательные значения $\Omega^{2}$. При $\xi<\xi_{k}$ низкосимметричное искажение- решетки можно формально считать «вмороженным» (поскольку $\left.\Omega^{2}>0\right)$, а фазовый переход для данного $t_{0}-$ локализованным при значении ведущего физического параметра, отвечающего $\xi_{k}$. При $t=0$, когда $\xi=\beta / \alpha, \Omega \sim W$ и зависимость $\Omega$ от этого параметра совпадает с зависимостью от него собственной вероятности переориентации. Такое положение может иметь место достаточно длительное время, пока $W t \ll 1^{* *}$. Проиллюстрируем сказанное для случая, когда $W$ определяется классичеоким выражением вероятностей надбарьерных. прыжков

$$
W(T)=v_{0} \exp \{-U / k T\}
$$

и можно говорить о температурных локальных ориентационных фазовых переходах. На рис. 1 показаны зависимости $\bar{Q}_{z 0} / Q_{z_{0}}$ от $T$ для различных времен $4 v_{0} t=10^{x}$ (взято $2 \beta=\alpha, U k^{-1}=400 \mathrm{~K}$ ). Естественно, что для времен, более близких к начальному, требуется более высокая температура для исчезновения анизотропных свойств. Для не слишком малых $x$ спад $\bar{Q}_{z 0}$ происходит достаточно резко. Поведение $\left(k_{\|}-k_{\perp}\right)$ аналогично.

Если собственная вероятность переориентации определяется туннелированием, возникает возможность наблюдать локальный фазовый переход под действием всестороннего сжатия [ $\left.{ }^{11}\right]$. Последнее обуслов-

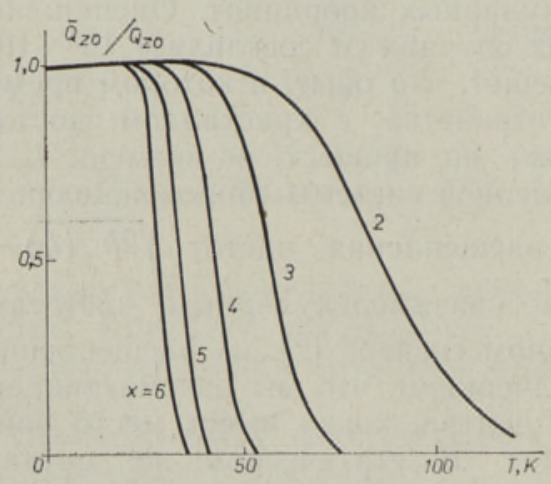

Рис. 1. Зависимость среднего низкосимметричного искажения решетки от температуры для различных времен $t=10^{x} / 4 v_{0}$.

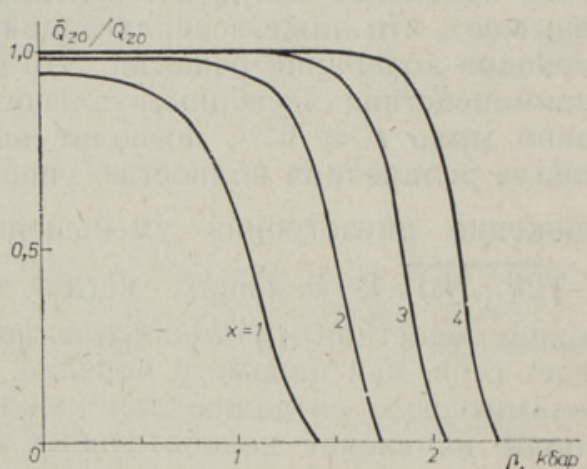

Рис. 2. Зависимость среднего низкосимметричного искажения решетки от всестороннего давления для различных времен $t=\left(10^{x} 4 W_{0}\right)^{-1}$.

** Расчет W составляет самостоятельную задачу, по которой выполнено много работ. Относительчо последних достижений см. [7-10]. 
ливает уменьшение соответствующего барьера и, в связи со свойствами потенциала (2), можно положить

$$
W(p)=W_{0} \exp \left(S p^{2}\right) .
$$

На рис. 2 приведены зависимости $\bar{Q}_{z 0} / Q_{z 0}$ от $p$ для различных времен $4 W_{0} t=10^{-x}$ (взято $\left.2 \beta=\alpha, S=2 \kappa б a p^{-2}\right)$. Для устранения анизотропии при меньших временах требуется большее давление. При больших временах заметно уменьшение $\bar{Q}_{z 0}$ уже в области малых $p$, как следствие туннелирования. Аналогично ведет себя $\left(k_{\|}-k_{\perp}\right)$. Зависимости от $p$, подобные изображенным на рис. 2 , наблюдались для внеузельных примесных центров, которые под действием всестороннего сжатия удавалось перевести в стабильную узельную конфигурацию [ $\left.{ }^{11}\right]$. Однако всестороннее сжатие влияет и на исходные параметры $Q_{z 0}, \alpha, \beta$, обращая их в пределе в нуль. Поэтому следует ожидать, что в данном случае резонансы, сопоставляемые $k_{\|}$и $k_{\perp}$, окажутся мягкими. Это означает, что с ростом давления частоты двух резонансов, сливаясь, будут стремиться к нулю. Но прежде чем они достигнут номинальної по $p$ точки локального фазового перехода, туннелирование приведет $k$ исчезновению анизотропных свойств.

Нам представляется, что вышесказанное иллюстрирует перспективность дальнейших экспериментов с различными вариациями временного разрешения в области явлений типа локальных релаксационных фазовых переходов.

Автор благодарит коллег, с которыми обсуждал рассматриваемый вопрос.

\section{ПРИЛОЖЕНИЕ}

Рассмотрим, например, второе из уравнений (11)

$$
\ddot{Q}_{\perp}(t)=-2 k_{\perp}(t) Q_{\perp}(t),
$$

представляющее собой задачу частотной модуляции. Представим его решение в виде

$$
Q_{\perp}(t)=A \exp i\left\{\omega\left(t_{0}\right) t-\int_{0}^{t} \Delta \omega\left(t^{\prime}\right) d t^{\prime}\right\}
$$

где

$$
\begin{aligned}
& \omega\left(t_{0}\right)=\left[2 / 3\left(\alpha+2 \beta-(\alpha-\beta) \mathrm{e}^{-6 W t_{0}}\right)\right]^{1 / 2}=\left[2 k_{\perp}\left(t_{0}\right)\right]^{1 / 2}, \\
& \Delta \omega(t)=\left(2(\alpha-\beta) / 3 \omega\left(t_{0}\right)\right)\left(\mathrm{e}^{-6 W t}-\mathrm{e}^{-6 W t_{0}}\right) .
\end{aligned}
$$

Решение (А2) удовлетворяет (A1), если $\left[\omega\left(t_{0}\right)\right]^{-2} \Delta \dot{\omega}(t) \ll 1$. Полагая $t=t_{0}+\Delta t$ и разлагая показатель экспоненты в (A2) до линейных по $\Delta t$ членов, находим

$$
Q_{\perp}(\Delta t)=A \exp i\left\{\omega\left(t_{0}\right) \Delta t+f\left(t_{0}\right)\right\},
$$

где $f\left(t_{0}\right)$ - нарастающий с $t_{0}$ сдвиг фазы. Указанное разложение по $\Delta t$ справедливо при условии $\Delta t \ll(6 W)^{-1}$, а аппроксимация решения формой (А2) возможна при аналогичном, но менее жестком условии. Следовательно, в интервале времени $\Delta t \ll W^{-1}$ около $t_{0}$ величина $Q_{\perp}$ совершает гармонические колебания с частотой $\omega\left(t_{0}\right)$. 


\section{ЛИТ Е РА Т У Р А}

1. Кр истофель Н. Н., Реб ане Л. А., Тр. ИФ АН ЭССР, № 48, 64-84 (1978).

2. Л ев анюк А. П., Осип ов В. В., Си гов А. С., Собянин А. А., Ж. эксперим. и теор. физики, 76, вып. $1,345-368$ (1979).

3. Кристофель Н. Н., Физ. твердого тела, 21, вып. 3, 895-901 (1979).

4. К ри ст офе ль Н. Н., Физ. твердого тела, 19, вып. 3, 775-780 (1977).

5. Кристофель Н. Н., Теория примесных центров малых радиусов в ионных кристаллах, М., «Наука», 1974.

6. Д ей ген М. Ф., Глиннчук М. Д., Успехи физ. наук, 114, № 2, 185-211 (1974).

7. W a g n e r, M., Solid State Commun., 18, № 9710, 1329-1331 (1976).

8. W a g n e r, M., Phys. status solidi (b), 88, № 2, 517-530 (1978).

9. D i ck, B. G., Phys. Rev., B 16, № 8, 3359-3370 (1977).

10. В и хнин В. С., Физ. твердого тела, 20, вып. 5, $1340-1346$ (1978).

11. Holl a nd, U., L üty, F., Ferroelectrics, 17, 377-379 (1977).

\section{Ннститут физики \\ Академии наук Эстонской ССР \\ Поступила в редакцию 2/IV 1979}

\section{N. KRISTOFFEL}

\section{ANISOTROOPSETE LISANDITSENTRITE LOKAALSETE RELAKSATSIOON-FAASISIIRETE ILLUSTRATSIOON}

On näidatud, et mitteinterakteeruvate anisotroopsete lisanditsentrite ümberorienteerumise tõenäosust muutva parameetri varieerimine võimaldab vaadelda lokaalse relaksatsioon-faasisiirde tüüpi nähtusi. Seejuures käituvad võremoonutus ja aktiivsetele võnkumistele vastava dünaamilise resonantsi löhenemine korrastuse parameetritena. On analüüsitud ajalisi efekte (anisotroopsuskao piirkonna sõltuvust katse sooritamise momendist).

\section{N. KRISTOFFEL}

\section{ILLUSTRATION OF LOCAL RELAXATIONAL PHASE TRANSITIONS IN ANISOTROPIC IMPURITY CENTRES}

In a simple model it is shown that altering the parameter that leads to the change of the re-orientation probability of anisotpopic non-interacting impurity centres, it is possible to observe the effects of the type of local relaxational phase transitions. The potential energy for the relaxing centre is calculated. The average lattice distortion shows the behaviour characteristic of the order parameter. For the time intervals of the interaction of the experimental device with the crystal, which are small as compared to the reciprocal re-orientational probability, it must be possible to detect the splitting of the dynamical resonance corresponding to active quasimolecular vibrations. This splitting also reflects the amount of the order in the system. Illustrative calculations are performed for the over-barrier-hopping (temperature transitions) and tunnelling (transitions under hydrostatic pressure) relaxations. Some timedependent effects are analyzed, like the shift of the region, where anisotropic properties vanish, with the change of the time at which the experiment starts after the relaxation of the fully-ordered state has begun. 